\title{
Reduced expression levels of let-7c in human breast cancer patients
}

\author{
XIN-XIN LI ${ }^{1 *}$, SHU-YAN GAO ${ }^{2 *}$, PING-YU WANG ${ }^{1}$, XUE ZHOU ${ }^{2}$, YOU-JIE LI $^{1}$, YUAN YU $^{1}$, \\ YUN-FEI YAN $^{1}$, HAN-HAN ZHANG ${ }^{1}$, CHANG-JUN LV ${ }^{2}$, HUI-HUI ZHOU ${ }^{3}$ and SHU-YANG XIE ${ }^{1}$ \\ ${ }^{1}$ Key Laboratory of Tumor Molecular Biology, Department of Biochemistry and Molecular Biology, \\ Binzhou Medical University; ${ }^{2}$ Department of Clinical Medicine, Affiliated Hospital of Binzhou Medical University, \\ Yantai, Shandong 264003; ${ }^{3}$ Department of Pathology, Affiliated Yuhuangding Hospital, \\ Medical College of Qingdao University, Yantai, Shandong 264000, P.R. China
}

Received May 1, 2014; Accepted December 16, 2014

DOI: $10.3892 / \mathrm{ol} .2015 .2877$

\begin{abstract}
Circulating microRNAs (miRNAs) are important in the diagnosis of a number of diseases, since serum or plasma miRNAs are more stable compared with miRNA isolated from blood samples. The aim of the present study was to investigate the association between the expression levels of serum let-7c miRNA and the clinical diagnosis of breast cancer (BC). The circulating let-7c levels of $90 \mathrm{BC}$ patients and 64 healthy controls were determined by performing a reverse transcription-quantitative polymerase chain reaction assay. The results demonstrated that let-7c expression was downregulated in the $\mathrm{BC}$ tissues compared with the paracarcinoma control tissues. In addition, the let-7c expression in the serum of $\mathrm{BC}$ patients was significantly lower compared with the healthy controls $(\mathrm{P}<0.01)$. Using a cutoff value of $0.374 \times 10^{3}$ copies $/ \mathrm{ml}$, the serum expression levels of let- $7 \mathrm{c}$ exhibited $87.5 \%$ sensitivity and $78.9 \%$ specificity for distinguishing $\mathrm{BC}$ patients from healthy controls (area under the receiver operating characteristic curve, $0.848 ; 95 \%$ confidence interval, 0.785-0.911). Furthermore, the results demonstrated that the serum expression levels of let-7c were significantly higher in premenopausal compared with postmenopausal
\end{abstract}

Correspondence to: Professor Shu-Yang Xie, Key Laboratory of Tumor Molecular Biology, Department of Biochemistry and Molecular Biology, Binzhou Medical University, 346 LaiShan GuanHai Road, Yantai, Shandong 264003, P.R. China E-mail: shuyangxie@aliyun.com

Abbreviations: miRNA, microRNA; RT-qPCR, reverse transcription-quantitative polymerase chain reaction; 3'-UTR, 3'-untranslated region; $\mathrm{BC}$, breast cancer; CI, confidence interval; $\mathrm{ER}$, estrogen receptor; PR, progesterone receptor; ROC, receiver operating characteristic; AUC, area under the ROC curve

*Contributed equally

Key words: circulating miRNA, breast cancer, let-7c, predictive factor, receiver operating characteristic analysis patients $(\mathrm{P}<0.05)$, supporting the hypothesis that postmenopausal status may affect the serum expression levels of let-7c. However, no statistically significant differences were detected in the serum levels of let-7c between ER (or PR)-positive and -negative patients. Therefore, the current study hypothesized that serum let-7c may be used as a novel and valuable biomarker for the diagnosis of $\mathrm{BC}$.

\section{Introduction}

MicroRNAs (miRNAs), a class of small and non-coding RNAs (length, 19-25 nucleotides), have the potential to regulate $\sim 30 \%$ of human genes, which may attribute to the development of cancer (1-3). miRNAs regulate gene expression by binding to the 3'-untranslated region (3'-UTR) of the targeted mRNAs. Numerous studies have indicated that abnormal expression of miRNA is associated with the development and progression of various types of human cancer (4-6). Although the biological function of miRNAs remains unknown, specific miRNAs have functions similar to tumor suppressors or oncogenes. Previous studies investigating the expression levels of miRNAs in cancer have indicated their significance and potential use in the classification of cancer or as diagnostic indicators $(5,7)$.

Breast cancer (BC) is one of the most common types of carcinoma and results in a high female mortality rate worldwide (1). In China, the number of BC-associated mortality rate has increased markedly in recent years $(3,7)$. Although early detection of $\mathrm{BC}$ is important in order to reduce the mortality rate of this disease, the methods available for primary detection, including mammography, ultrasonography and magnetic resonance imaging, may result in misdiagnosis or missed diagnosis. The expression pattern of each miRNA molecule varies in different cancer phenotypes (including BC) and, thus, can be used in tumor classification, diagnosis, therapy and prognosis (4). Over the past decades, the association between miRNAs and human BC has been extensively investigated (8-10). Previous studies have demonstrated that the expressional changes of a number of miRNAs are involved in BC development and progression $(2,5)$, while serum or plasma miRNA expression levels were found to be different in $\mathrm{BC}$ 
patients and healthy controls (3). Therefore, miRNAs represent a novel approach to BC diagnosis.

The present study aimed to further investigate the association between serum miRNA expression levels and the clinical diagnosis of $\mathrm{BC}$. The circulating let-7c levels were determined in the serum of $\mathrm{BC}$ patients and healthy controls by performing individual-based reverse transcription-quantitative polymerase chain reaction (RT-qPCR) assays, in accordance with previous reports $(2,3,11)$. In addition, the present study investigated whether the serum let-7c level can be used as a potential biomarker for $\mathrm{BC}$ diagnosis.

\section{Materials and methods}

Study subjects. The present study was conducted in the Inpatient Department of Medical Oncology at the Affiliated Hospital of Binzhou Medical University (Yantai, China) and performed in accordance with the relevant guidelines of the Medical Ethics Committee of Binzhou Medical University.

In total, 90 females with BC (age range, 27-79 years), who were pathologically diagnosed with $\mathrm{BC}$ for the first time between June 1st 2010 and July 31st 2013, were included in the present study. The patients had not been previously treated with chemotherapy or postmenopausal hormone therapy. In addition, 64 healthy controls were recruited from individuals who visited the Affiliated Hospital of Binzhou Medical University for physical examination within the same time period and were not diagnosed with a tumor or physical illness. Prior to enrollment in the present study, written informed consent was obtained from each individual.

Immunohistochemistry. Fresh BC and healthy tissues from patients who underwent surgery at the Affiliated Hospital of Binzhou Medical University were obtained at the time of surgery, and immediately prepared for pathological diagnosis, immunohistochemistry or RNA isolation. The cancer sample sections containing a minimum of $60 \%$ cancer cells were used in the experiments. All patients provided their written consent to participate in this study. Subsequently, the tissues were fixed in $4 \%$ paraformaldehyde, embedded in paraffin and cut into $5-\mu \mathrm{M}$ sections. Subsequently, the sections were deparaffinized in xylene, rehydrated and antigen retrieval was performed by incubation in $10 \mathrm{mM}$ citrate buffer, $\mathrm{pH} 6.0$ at $95-100^{\circ} \mathrm{C}$ for $10 \mathrm{~min}$. This was followed by washing in phosphate-buffered saline (PBS). To quench the endogenous peroxidase activity, the slides were incubated in $3 \%$ hydrogen peroxide for $15 \mathrm{~min}$ at room temperature. Next, the BC slides were incubated in $10 \%$ normal goat serum [Beijing Zhongshan Golden Bridge Biotechnology Co., Ltd., Beijing, China] for $20 \mathrm{~min}$ at room temperature to prevent non-specific staining. Subsequently, the slides were incubated at $4^{\circ} \mathrm{C}$ overnight with appropriate dilutions of the following primary antibodies: Rabbit monoclonal anti-estrogen receptor (ER; 1:200); and rabbit monoclonal anti-progesterone receptor (PR; 1:200; Beijing Zhongshan Golden Bridge Biotechnology Co., Ltd.). Next, the samples were incubated with horseradish peroxidase-conjugated goat anti-rabbit secondary antibodies (1:5000, Beijing Zhongshan Golden Bridge Biotechnology Co., Ltd.). The negative controls were incubated in $1 \mathrm{X}$ PBS without antibody, following the same procedure. The samples was visualized using an ABC kit (Beijing Zhongshan Golden Bridge Biotechnology Co., Ltd.) and positive ER and PR status was considered when nuclear staining was $>10 \%$. The expression of ER and PR was examined under the Olympus BX51 AX-70 microscope (Olympus, Tokyo, Japan).

Sample collection. Whole blood samples $(3 \mathrm{ml})$ were obtained from each subject. The serum samples were separated by centrifugation at 2,650 $\mathrm{x}$ g for $10 \mathrm{~min}$ at room temperature and stored at $-80^{\circ} \mathrm{C}$ prior to analysis (11).

miRNA isolation from serum or tissue samples. miRNA was extracted from the serum samples using the mirVana ${ }^{\mathrm{TM}}$ miRNA isolation kit (Ambion Life Technologies, Carlsbad, CA, USA) according to the manufacturer's instructions. The $\mathrm{BC}$ tissues were homogenized in a denaturing lysis solution and treated with TRIzol reagent (Invitrogen Life Technologies, Carlsbad, CA, USA) to extract total RNA, according to the manufacturer's instructions. Subsequently, the mirVana ${ }^{\mathrm{TM}}$ miRNA isolation kit was used to obtain the miRNAs from 30-50 mg total RNA samples.

$R T$-qPCR. Poly(A) tails were added to the extracted miRNAs using poly(A) polymerase (Ambion Life Technologies) and the complementary (c)DNA molecules were synthesized using the 5'-AACATGTACAGTCCATGG ATGd(T)30N(A, G, C or T)-3' primer. RT-qPCR of Let-7c was performed using the following primers: Forward, 5'-GGTTGA GGTAGTAGGTTGTATGGT-3'; and reverse, 5'-AACATG TACAGTCCATGGATG-3'. Each RT-qPCR reaction mixture contained $0.5 \mu \mathrm{l}$ cDNA, $7.5 \mu 1$ sterile water, $1 \mu 1$ forward primer, $1 \mu \mathrm{l}$ reverse primer and $10 \mu \mathrm{l}$ of $\mathrm{SYBR}^{\circledR}$ Premix Ex Taq $^{\text {TM }}$ (Takara Biotechnology Co., Ltd., Dalian, China) and was performed using the Rotor-Gene 3000 system (Corbett Life Science, Mortlake, Australia) as follows: Initial denaturation at $95^{\circ} \mathrm{C}$ for $5 \mathrm{~min}$, followed by 40 cycles of denaturation at $95^{\circ} \mathrm{C}$ for $20 \mathrm{sec}$, annealing at $60^{\circ} \mathrm{C}$ for $20 \mathrm{sec}$ and extension at $72^{\circ} \mathrm{C}$ for $30 \mathrm{sec}$. As described in previous studies, $5 \mathrm{~S}$ ribosomal RNA was used as the reference control $(12,13)$. All the experiments were performed in triplicate.

Statistical analysis. The experimental data were initially analyzed for normal distribution and variance homogeneity using the Shapiro-Wilk test and F-test, respectively. Normal distribution data are represented as the mean \pm standard deviation, and all the other data are represented as median and quartiles. Differences in the age, height or weight between the BC patients and healthy controls were analyzed using the Student's t-test. However, when the serum expression levels of let-7c did not demonstrate normal distribution, nonparametric tests were applied to analyze these differences. In addition, continuous variables between the $\mathrm{BC}$ patients and controls were analyzed by performing the Wilcoxon rank-sum test. Statistical analyses were performed using $\mathrm{R}^{\odot}$ software (version 2.15.0; http://www.r-project.org). $\mathrm{P}<0.05$ was considered to indicate a statistically significant difference. Furthermore, receiver operating characteristic (ROC) curves were generated using the SPSS software (version 18.0; SPSS, Inc., Chicago, IL, USA) to assess the diagnostic accuracy of each parameter. The area under the ROC curve (AUC) was used to identify 
Table I. Demographic and clinical characteristics of the study samples.

\begin{tabular}{|c|c|c|c|}
\hline Parameter & Healthy controls $(n=64)$ & Patients $(n=90)$ & P-value \\
\hline Age, years ${ }^{b}$ & $43.781 \pm 15.831$ & $47.900 \pm 9.882$ & 0.068 \\
\hline Weight, $\mathrm{kg}^{\mathrm{b}}$ & $62.638 \pm 8.844$ & $65.408 \pm 8.238$ & 0.090 \\
\hline Height, $\mathrm{cm}^{\mathrm{b}}$ & $160.500 \pm 6.414$ & $158.551 \pm 4.619$ & 0.075 \\
\hline Estrogen receptor-positive/negative, $\mathrm{n}$ & - & $64 / 26$ & - \\
\hline Progesterone receptor-positive/negative, $\mathrm{n}$ & - & $60 / 30$ & - \\
\hline Premenopausal/postmenopausal, $\mathrm{n}$ & $35 / 29$ & $48 / 42$ & 0.998 \\
\hline Median let- $7 \mathrm{c}, \mathrm{x} 10^{3}$ copies $/ \mathrm{ml}$ & 2.300 & 0.035 & $<0.01$ \\
\hline
\end{tabular}

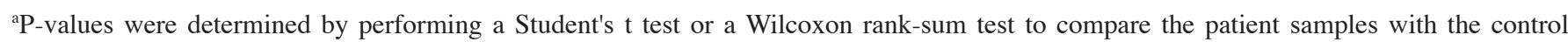

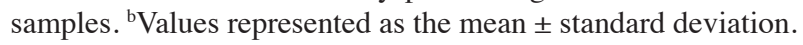

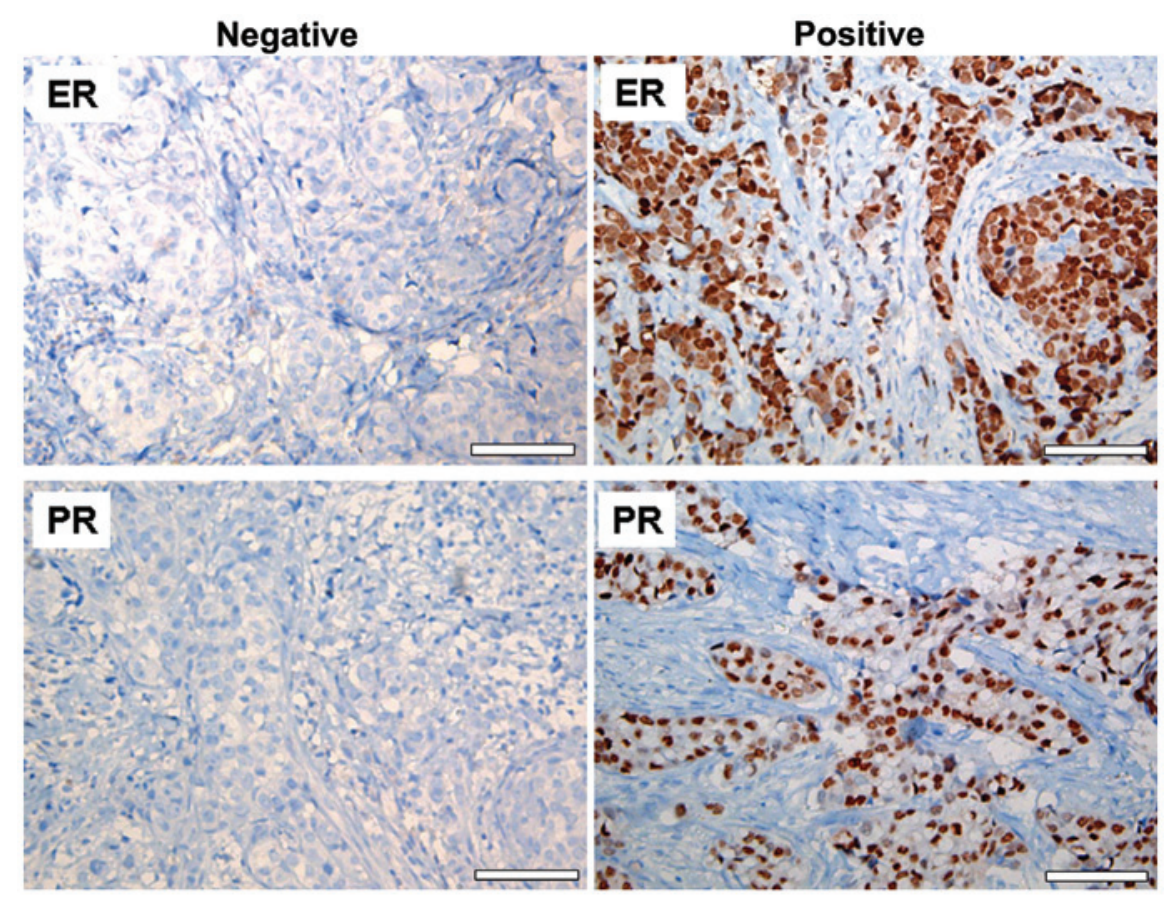

Figure 1. Immunohistochemical analysis of estrogen receptor (ER) and progesterone receptor (PR) expression levels in breast cancer tissues. Using a limit of $>10 \%$ nuclear staining to define positive ER and PR status, 64 patients were determine as ER-positive and 60 patients as PR-positive. Scale bar, $150 \mu \mathrm{m}$.

the optimal sensitivity and specificity levels at which cancer patients can be distinguished from healthy individuals.

\section{Results}

Clinical characteristics. The demographic and clinical characteristics of the $90 \mathrm{BC}$ patients and 64 healthy controls that participated in the present study are listed in Table I. No statistically significant differences in the age, height or weight were identified between the BC patients and healthy controls. In addition, no clear statistically significant differences in the menopausal status were identified between the BC patients and healthy controls.

The presence of ER and PR in the BC tissue samples was detected by immunohistochemical analysis. Nuclear staining of $>10 \%$ was considered to indicate positivity ER and PR status (Fig. 1). Of the 90 BC patients, 64 patients were ER-positive $(71.1 \%)$ and 60 were PR-positive $(66.6 \%)$.
Reduced let-7c expression levels in BC tissues. Considerable evidence accumulated from a number of previous studies has indicated that downregulation of let-7 family miRNAs may be associated with a poor clinical outcome in BC patients $(14,15)$. Therefore, to investigate the role of let-7c in $\mathrm{BC}$, the present study analyzed the expression levels of let-7c in BC tissues. The results indicated that let-7c expression was markedly decreased ( $>20$-fold lower) in BC tissues $(n=4)$ compared with paracarcinoma tissues ( $n=4$; Fig. $2 A$ ), supporting the suppressive role of let-7c in tumor proliferation.

Reduced serum let-7c expression levels in BC patients. The serum expression levels of let-7c were detected by performing qPCR analysis to investigate the potential role of let-7c in the diagnosis of $\mathrm{BC}$. The results of the present study demonstrated that serum let-7c levels in $\mathrm{BC}$ patients $\left(0.035 \times 10^{3}\right.$ copies $/ \mathrm{ml}$; $\mathrm{n}=90$ ) were significantly lower compared with the healthy controls $\left(2.300 \times 10^{3}\right.$ copies $/ \mathrm{ml} ; \mathrm{n}=64 ; \mathrm{P}<0.01$; Table I; Fig. 2B). 
Table II. Association of ER, PR and menopausal status with let-7c.

\begin{tabular}{|c|c|c|}
\hline Parameter & $\begin{array}{l}\text { Median let- } 7 \mathrm{c} \\
\mathrm{x} 10^{3} \text { copies } / \mathrm{ml}\end{array}$ & P-value ${ }^{a}$ \\
\hline ER & & 0.541 \\
\hline Positive ( $n=64)$ & 0.033 & \\
\hline Negative $(n=26)$ & 0.036 & \\
\hline PR & & 0.986 \\
\hline Positive $(n=60)$ & 0.035 & \\
\hline Negative $(n=30)$ & 0.033 & \\
\hline Menopausal status & & $0.040^{\mathrm{a}}$ \\
\hline Premenopausal $(\mathrm{n}=48)$ & 0.036 & \\
\hline Postmenopausal $(n=42)$ & 0.032 & \\
\hline
\end{tabular}

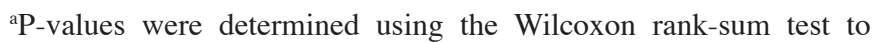
compare premenopausal samples with postmenopausal samples. ER, estrogen receptor; PR, progesterone receptor.

$\mathbf{A}$

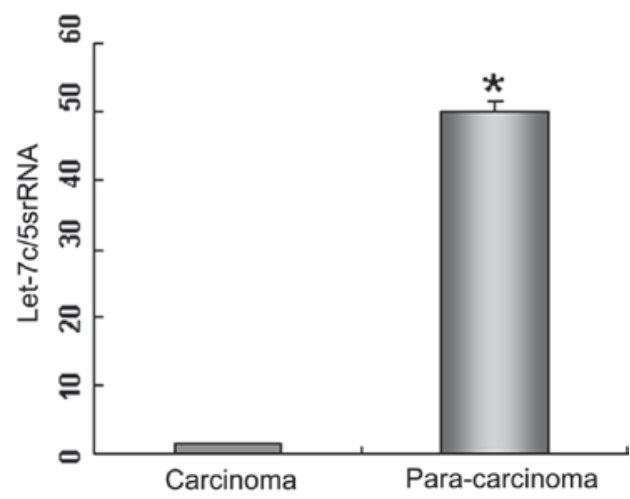

B

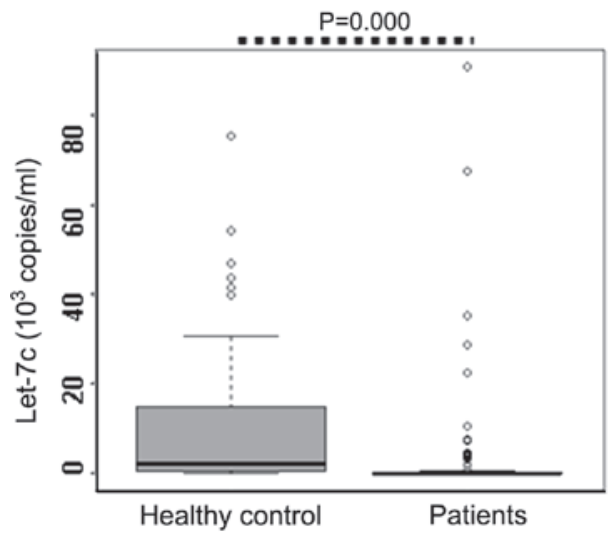

Figure 2. Detection of let-7c expression levels in (A) tissue samples, using reverse transcription-quantitative polymerase chain reaction, and (B) serum samples from breast cancer (BC) patients and heathy controls. let-7c expression was significantly lower in the $\mathrm{BC}$ tissues compared with the paracarcinoma tissues $\left(\mathrm{n}=4 ;{ }^{*} \mathrm{P}<0.01\right.$ vs. carcinoma; control, $5 \mathrm{~S}$ ribosomal RNA). In addition, the serum let-7c expression levels were lower in BC patients $(n=90)$ compared with the healthy controls $(n=64 ; P=0.000)$. Data are expressed as the mean \pm standard deviation.

These results were consistent with the let-7c expression levels identified in the $\mathrm{BC}$ tissues samples, indicating that let-7c may be an important factor in $\mathrm{BC}$ diagnosis.

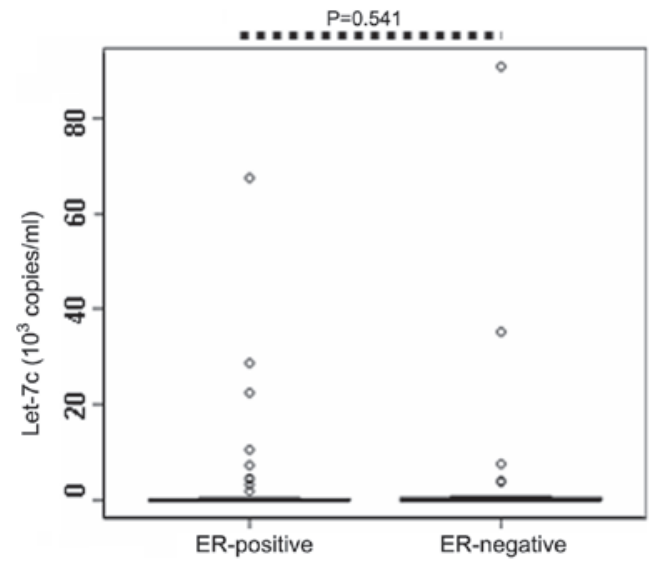

Figure 3. Correlation between serum let-7c expression levels and ER-positive expression in breast cancer (BC) patients. The serum let-7c levels in ER-positive $B C$ patients $(n=64)$ were not significantly different when compared with the ER-negative $\mathrm{BC}$ patients $(\mathrm{n}=26 ; \mathrm{P}=0.541)$. ER, estrogen receptor; PR, progesterone receptor.

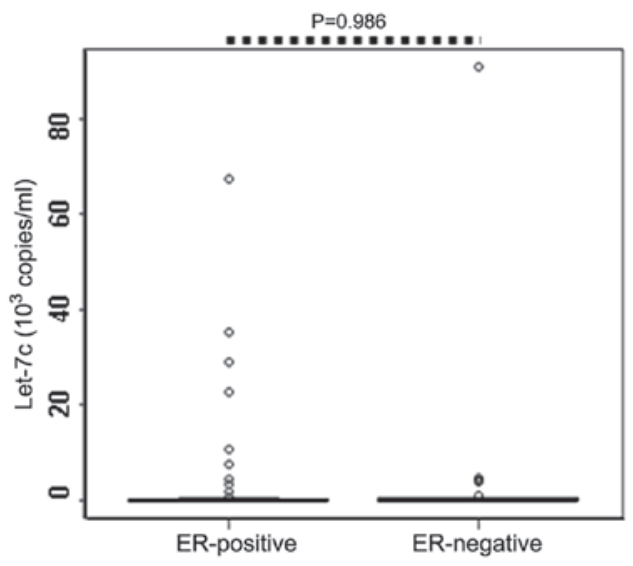

Figure 4. Correlation between serum let-7c expression levels and PR-positive expression in breast cancer (BC) patients. No significant difference was identified between the serum let-7c expression levels of the PR-positive $(n=60)$ and $\mathrm{PR}$-negative $\mathrm{BC}$ patients $(\mathrm{n}=30 ; \mathrm{P}=0.986)$. $\mathrm{PR}$, progesterone receptor; $\mathrm{ER}$, estrogen receptor.

Correlation between ER/PR status and circulating let-7c expression levels. ER and PR have been previously reported as important factors associated with the etiology and therapeutic treatment strategy of BC $(16,17)$. Therefore, to investigate the correlation between ER or PR status and the serum expression levels of let-7c, let-7c expression was compared between ER- (or PR-) positive and negative patients. The results demonstrated that serum expression levels of let-7c in the ER-positive patients $\left(n=64 ; 0.033 \times 10^{3}\right.$ copies $\left./ \mathrm{ml}\right)$ were not significantly different compared with the ER-negative patients $(n=26$; $0.036 \times 10^{3}$ copies $/ \mathrm{ml}$; P=0.541; Table II; Fig. 3). Similarly, no statistically significant difference was identified between the serum levels of let-7c in the PR-positive $(n=60)$ and PR-negative patients ( $\mathrm{n}=30$; $\mathrm{P}=0.986$; Table II; Fig. 4).

Correlation between menopausal status and circulating let-7c expression levels. Since postmenopausal females with a high breast density exhibit increased risk of developing BC (18), the serum let-7c expression levels were compared 


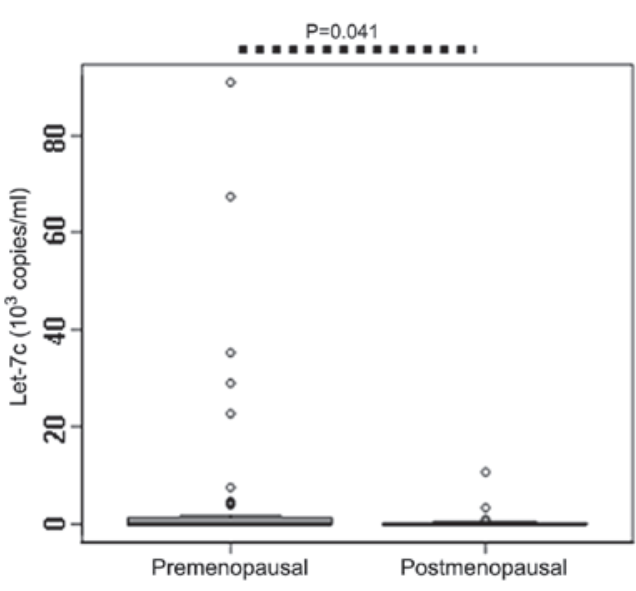

Figure 5. Correlation between serum let-7c levels and menopausal status of breast cancer patients. The serum let-7c levels in premenopausal status patients $(n=48)$ were evidently higher compared with those in postmenopausal status patients $(n=42 ; P=0.040)$.

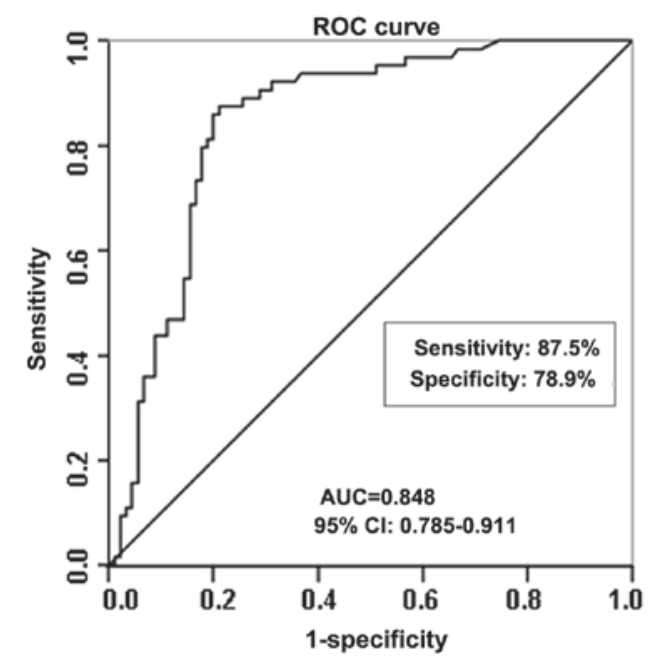

Figure 6. ROC curves for serum let-7c expression levels in breast cancer (BC) patients, indicating an AUC with the diagnostic power to distinguish BC patients from healthy controls. ROC, receiver operating characteristic; AUC, area under the ROC curve; CI, confidence interval.

between premenopausal and postmenopausal patients. The results identified that the expression levels of let-7c in the premenopausal patients $\left(0.036 \times 10^{3}\right.$ copies $\left./ \mathrm{ml}\right)$ was significantly higher compared with the postmenopausal patients $\left(0.032 \times 10^{3}\right.$ copies/ml; P=0.040; Table II; Fig. 5), which indicates that the postmenopausal status may affect the expression level of serum let-7c.

Diagnostic potential of let-7c expression levels in BC. Statistical ROC analysis was used to investigate the diagnostic potential of let-7c serum expression levels in $\mathrm{BC}$ patients. The expression data for let-7c were plotted using the ROC curves to identify a cut-off value that could distinguish breast cancer patients from healthy controls. Using a cutoff level of $0.374 \times 10^{3}$ copies $/ \mathrm{ml}$, the serum expression levels of let-7c presented sensitivity of $87.5 \%$ and specificity of $78.9 \%$ in distinguishing the $\mathrm{BC}$ patients from the healthy controls, with an AUC of 0.848 (95\% confidence interval, 0.785-0.911; $\mathrm{P}<0.001$; Fig. 6).

\section{Discussion}

let-7 miRNAs are members of a highly conserved miRNA family consisting of 12 genes (including let-7-a1, -a2, -a3, -b, -c, -d, -e, -f1, -f2, -g, -i and miR-98), which are located on eight different chromosomes (19). Let-7 was initially described in Caenorhabditis elegans and functionally conserved from lower invertebrates to higher mammals (20). In the present study, a comprehensive investigation of serum let-7c miRNA expression was conducted in BC patients and healthy controls using RT-qPCR (21). The expression levels of let-7c were significantly decreased in the serum of the $\mathrm{BC}$ patients compared with the healthy controls, which indicates that serum let-7c may have a considerable diagnostic function in differentiating BC patients from healthy controls.

The let-7 family members function as tumor suppressors and have been associated with various target genes, including Ras (20), high mobility group AT-hook $2(22,23)$ and B-cell lymphoma-extra large (Bcl-xL) (24). let-7 expression is downregulated in a number of malignancies. For instance, let-7 was identified to be decreased in human hepatoma cells and tissues, which are associated with enhanced expression of Bcl-xL (24). A previous study revealed that let-7c was a downregulated epithelial miRNA and its functions were delineated in unique cells derived from columnar cell hyperplasia (25). Similarly, the present study identified that let-7c expression was downregulated in $\mathrm{BC}$ tissues compared with paracarcinoma tissues. Thus, the present and aforementioned studies indicated the suppressive role of let-7 miRNAs in tumorigenesis.

A greater number of studies focusing on the function of miRNAs have been conducted, particularly those investigating the roles of circulating miRNAs in disease diagnosis (20). Circulating miRNAs isolated from the serum or plasma are more stable compared with those isolated from the blood (10). In addition, circulating miRNAs are stable at room temperature and can survive under the effects of RNase and DNase $(1,11)$. Thus, the expression patterns of circulating miRNAs may be used as potential diagnostic indicators for a number of diseases, including tumors, improving cancer diagnosis. To further investigate the role of let-7c expression levels in the diagnosis of $\mathrm{BC}$, the circulating let-7c levels were compared between $\mathrm{BC}$ patients and healthy controls. It was identified that let-7c expression was lower in the serum of $\mathrm{BC}$ patients compared with the healthy controls. Furthermore, at the optimal cut-off, the serum level of let-7c exhibited sensitivity of $87.5 \%$ and specificity of $78.9 \%$ for distinguishing BC patients from healthy controls.

The important role of pathological analysis in the diagnosis of $\mathrm{BC}$ is biomarker testing, specifically the accurate assessment of the ER and PR status of BC tissues $(26,27)$. Previously, significant associations have been reported between tumor size (or the presence of distant metastases) in BC and ER/PR-positive rate (or menopausal status) (28). Therefore, the association between the expression level of let-7c and the ER/PR-positive rate, as well as the menopausal status of the patients, was investigated in the present study. The results indicated that $71.1 \%$ of primary BC patients expressed ER, while 66.6\% expressed PR. Subsequent investigation into the association between ER (or PR)-positive expression and serum expression levels of let-7c revealed that ER (or PR)-positive expression did not affect 
the serum expression levels of let-7c. However, let-7c expression in the premenopausal patients was significantly higher compared with the postmenopausal patients, indicating that the postmenopausal status may affect let-7c expression levels. Moreover, Kerlikowske et al reported that postmenopausal females with a high breast density presented an increased risk of developing BC (18).

In conclusion, the present study identified that let-7c expression was lower in $\mathrm{BC}$ tissues compared with paracancerous tissues. Furthermore, the let-7c serum levels of the $\mathrm{BC}$ patients were significantly lower compared with the levels of the healthy controls and were affected by the menopausal status of the patients. Although the results of the current study indicate that serum let-7c expression levels may represent a novel diagnostic biomarker for BC patients, well-designed studies with larger sample sizes are required to further confirm the role of let-7c in cancer diagnosis.

\section{Acknowledgements}

This study was supported by grants from the National Natural Science Foundation (nos. 31371321, 31440061 and 81200601), NCET-10-0919, the Taishan Scholars program of Shandong Province, the Shandong Science and Technology Committee (no. ZR2012HQ035) and the Foundation of Shandong Educational Committee (no. J11LC01).

\section{References}

1. Asaga S, Kuo C, Nguyen T, et al: Direct serum assay for microRNA-21 concentrations in early and advanced breast cancer. Clin Chem 57: 84-91, 2011.

2. Heneghan HM, Miller N, Lowery AJ, et al: MicroRNAs as novel biomarkers for breast cancer. J Oncol 2009: 950201, 2009.

3. Roth C, Rack B, Müller V, et al: Circulating microRNAs as blood-based markers for patients with primary and metastatic breast cancer. Breast Cancer Res 12: R90, 2010.

4. Macfarlane LA and Murphy PR: MicroRNA: Biogenesis, function and role in cancer. Curr Genomics 11: 537-561, 2010.

5. Zhao B, Han H, Chen J, et al: MicroRNA let-7c inhibits migration and invasion of human non-small cell lung cancer by targeting ITGB3 and MAP4K3. Cancer Lett 342: 43-51, 2014.

6. Sempere LF, Freemantle S, Pitha-Rowe I, et al: Expression profiling of mammalian microRNAs uncovers a subset of brain-expressed microRNAs with possible roles in murine and human neuronal differentiation. Genome Biol 5: R13, 2004.

7. Pelosi A, Careccia S, Lulli V, et al: miRNA let-7c promotes granulocytic differentiation in acute myeloid leukemia. Oncogene 32: 3648-3654, 2013.

8. Qian B, Katsaros D, Lu L, et al: High miR-21 expression in breast cancer associated with poor disease-free survival in early stage disease and high TGF-beta1. Breast Cancer Res Treat 117: 131-140, 2009.

9. Wang PY, Sun YX, Zhang S, et al: Let-7c inhibits A549 cell proliferation through oncogenic TRIB2 related factors. FEBS Lett 587: 2675-2681, 2013.
10. Wu Q, Wang C, Lu Z, Guo L and Ge Q: Analysis of serum genome-wide microRNAs for breast cancer detection. Clin Chim Acta 413: 1058-1065, 2012.

11. Chen X, Ba Y, Ma L, et al: Characterization of microRNAs in serum: a novel class of biomarkers for diagnosis of cancer and other diseases. Cell Res 18: 997-1006, 2008.

12. Zhang HH, Pang M, Dong W, et al: miR-511 induces the apoptosis of radioresistant lung adenocarcinoma cells by triggering BAX. Oncol Rep 31: 1473-1479, 2014.

13. Wang PY, Sun YX, Zhang S, et al: Let-7c inhibits A549 cell proliferation through oncogenic TRIB2 related factors. FEBS Lett 587: 2675-2681, 2013.

14. Hu X, Guo J, Zheng L, et al: The heterochronic microRNA let-7 inhibits cell motility by regulating the genes in the actin cytoskeleton pathway in breast cancer. Mol Cancer Res 11: 240-250, 2013.

15. Piskounova E, Polytarchou C, Thornton JE, et al: Lin28A and Lin28B inhibit let-7 microRNA biogenesis by distinct mechanisms. Cell 147: 1066-1079, 2011

16. Cui X, Schiff R, Arpino G, Osborne CK and Lee AV: Biology of progesterone receptor loss in breast cancer and its implications for endocrine therapy. J Clin Oncol 23: 7721-7735, 2005.

17. Diaz NM: Laboratory testing for HER2/neu in breast carcinoma: an evolving strategy to predict response to targeted therapy. Cancer Control 8: 415-418, 2001.

18. Kerlikowske K, Cook AJ, Buist DS, et al: Breast cancer risk by breast density, menopause and postmenopausal hormone therapy use. J Clin Oncol 28: 3830-3837, 2010.

19. Qin B, Xiao B, Liang D, et al: MicroRNA let-7c inhibits Bcl-xl expression and regulates ox-LDL-induced endothelial apoptosis. BMB Rep 45: 464-469, 2012.

20. Johnson SM, Grosshans H, Shingara J, et al: RAS is regulated by the let-7 microRNA family. Cell 120: 635-647, 2005.

21. Jones CI, Zabolotskaya MV, King AJ, et al: Identification of circulating microRNAs as diagnostic biomarkers for use in multiple myeloma. Br J Cancer 107: 1987-1996, 2012.

22. Sakurai M, Miki Y, Masuda M, et al: LIN28: a regulator of tumor-suppressing activity of let-7 microRNA in human breast cancer. J Steroid Biochem Mol Biol 131: 101-106, 2012.

23. Zhu XM, Wu LJ, Xu J, Yang R and Wu FS: Let-7c microRNA expression and clinical significance in hepatocellular carcinoma. J Int Med Res 39: 2323-2329, 2011.

24. Shimizu S, Takehara T, Hikita $\mathrm{H}$, et al: The let-7 family of microRNAs inhibits Bcl-xL expression and potentiates sorafenib-induced apoptosis in human hepatocellular carcinoma. J Hepatol 52: 698-704, 2010.

25. Björner S, Fitzpatrick PA, Li Y, et al: Epithelial and stromal microRNA signatures of columnar cell hyperplasia linking Let-7c to precancerous and cancerous breast cancer cell proliferation. PLoS One 9: e105099, 2014.

26. Barnes DM and Hanby AM: Oestrogen and progesterone receptors in breast cancer: past, present and future. Histopathology 38 : 271-274, 2001.

27. Sofi GN, Sofi JN, Nadeem R, et al: Estrogen receptor and progesterone receptor status in breast cancer in relation to age, histological grade, size of lesion and lymph node involvement. Asian Pac J Cancer Prev 13: 5047-5052, 2012.

28. Faheem M, Mahmood H, Khurram M, Qasim U and Irfan J: Estrogen receptor, progesterone receptor and Her $2 \mathrm{Neu}$ positivity and its association with tumour characteristics and menopausal status in a breast cancer cohort from northern Pakistan. Ecancermedicalscience 6: 283, 2012. 